\title{
Retraction Note: Retraction note to: The smell of fear: on the relation between test smells and flaky tests
}

\author{
Fabio Palomba ${ }^{1}$ (D) Andy Zaidman ${ }^{2}$ \\ Published online: 26 March 2020 \\ (C) Springer Science+Business Media, LLC, part of Springer Nature 2020
}
Retraction note to: Empirical Software Engineering (2019) 24:2907-2946. https://doi.org/10.1007/s10664-019-09683-z

The authors have retracted this article Palomba and Zaidman (2019). Upon re-review of the experiment presented in the article, the authors identified errors in the flaky test detection strategy. After careful analysis of the replication study, the results presented in this article are rendered unreliable. All authors agree to this retraction.

\section{Reference}

Palomba F, Zaidman A (2019) The smell of fear: on the relation between test smells and flaky tests. Empir Software Eng 24:2907-2946. https://doi.org/10.1007/s10664-019-09683-z

The online version of the original article can be found at https://doi.org/10.1007/s10664-019-09683-z

Fabio Palomba

palomba@ifi.uzh.ch

Andy Zaidman

a.e.zaidman@tudelft.nl

1 University of Zurich, Zurich, Switzerland

2 Delft University of Technology, Delft, The Netherlands 